\title{
Circular Motion Geometry Using Minimal Data
}

\author{
Guang JIANG ${ }^{1}$, Long QUAN ${ }^{2}$, and Hung-tat TSUI ${ }^{1}$ \\ ${ }^{1}$ Dept. of Electronic Engineering, The Chinese University of Hong Kong \\ ${ }^{2}$ Dept. of Computer Science, The Hong Kong University of Science and Technology \\ gjiang,httsui@ee.cuhk.edu.hk, quan@cs.ust.hk
}

\begin{abstract}
Circular motion or single axis motion is widely used in computer vision and graphics for 3D model acquisition. This paper describes a new and simple method for recovering the geometry of uncalibrated circular motion from a minimal set of only two points in four images. This problem has been previously solved using non-minimal data either by computing the fundamental matrix and trifocal tensor in three images, or by fitting conics to tracked points in five or more images. It is first established that two sets of tracked points in different images under circular motion for two distinct space points are related by a homography. Then, we compute a plane homography from a minimal two points in four images. After that, we show that the unique pair of complex conjugate eigenvectors of this homography are the image of the circular points of the parallel planes of the circular motion. Subsequently, all other motion and structure parameters are computed from this homography in a straighforward manner. The experiments on real image sequences demonstrate the simplicity, accuracy and robustness of the new method.
\end{abstract}

Key words: structure from motion, minimal data, turntable, circular motion, vision geometry, single axis motion. 


\section{Introduction}

Acquiring 3D models from circular motion sequences, particularly turntable sequences, has been widely used by computer vision and graphics researchers $[3,18,23,24]$ as this method is very simple and robust. Generally, the whole reconstruction procedure includes, first, the determination of camera positions at different viewpoints, or equivalently, the different positions of the rotating device; second, the detection of object boundaries as silhouttes; and, third, the extraction of a visual hull as the surface model from a volume representation. Other more detailed surface models can also be computed from the known geometry of the circular motion [13]. The reliable estimation of the camera positions or the rotation angles relative to a static camera are the most important and difficult part of this kind of modeling system. Traditionally or in many commercial systems, rotation angles are often controlled by an electro-mechanical system or obtained by careful calibration procedures [18, 23, 24]. Fitzgibbon et al. [8] extended the analysis of the circular motion to be able to recover unknown rotation angles from uncalibrated image sequences based on a projective geometry approach and multi-view geometric constraints. Corresponding points are tracked for each pair of images for the fundamental matrices and for each triplet of images for the trifocal tensors. Jiang et al. [11] recently developed new methods to compute single axis motion by fitting the conic locus of the tracked points in at least five images. Another interesting approach is proposed by Mendonça et al. [16, 17] who recovered the circular motion by using surface profiles.

The approach based on conic fitting [11] is obviously less general than the method [8] based on multi-view constraints, but it naturally integrates the geometric constraints of motion circularity directly into the analysis process and it does reveal the intrinsic properties of circular motion geometry. However, by counting invariant quantities under circular motion, it is evident that all existing methods use excessively redundant data points, i.e., the existing solutions are not minimal. In this paper, we propose a new method that requires only as few as two points in four images. Furthermore, the algorithm for the new method is remarkably simple. First, we estimate a planar homographical transform between two points tracked in at least four images; then, we calculate the eigenvectors of this $3 \times 3$ transformation to obtain the invariant quantities of the ciruclar motion. The advantage of this new method over existing methods is straightforward. First, it uses only a total of 8 points (2 points

in 4 images), while the existing methods need either at least 21 points (7 points in 3 images) [8] or 
10 points ( 2 points in 5 images) [11]. Second, the calculation is presented in a closed-form solution obtained by solving only a real cubic equation. Finally, it is well-known in geometric computation in computer vision that developing efficient algebraic minimal methods is of primary importance, both for automatically computing correspondences and for final optimal estimation of the geometry $[6,10]$. A short version of this paper is published in the ICCV conference [12].

The paper is organized as follows. Section 2 reviews the invariants under circular motion, Section 3 gives more technical details of the existing methods and analyzes their insufficiency. These two sections motivate and prepare for the presentation of the new method using minimal data in Section 4. Analysis and proof of the results used in the new method are given in Section 5. A RANSAC-driven method is presented in Section 6 to choose the most reliable points and optimize the results for a complete modeling system. Section 7 demonstrates the validity of the approach using intensive experiments. A short conclusion is presented in Section 8.

\section{Review of circular motion and its associated fixed image enti- ties}

Circular motion or single axis motion consists of a set of Euclidean actions such that the relative motion between a scene and a camera can be described by the rotation about a single fixed axis. The most common case is a static camera that views an object that is rotating on a turntable with fixed internal parameters [18]. The equivalent case is when an object is fixed and the camera rotates around it. This is a particular case of the more general planar motion $[1,5]$ as it restricts all motion to rotation around a single axis. Without loss of generality, we assume that the rotation axis is vertical as the $z$-axis of the world coordinates and the camera is moving on the horizontal plane.

The fixed image entities of the circular motion are similar to planar motion, which includes two lines. One is the image of the rotation axis, $\mathbf{l}_{s}$. Note that $\mathbf{l}_{s}$ is a line of fixed points. Unlike in planar motion, line $\mathbf{l}_{s}$ is fixed in all images under circular motion. The other line is called the horizon line, $\mathbf{l}_{h}$. It is the image of the vanishing line of the horizontal plane. Unlike the image of the rotation axis, the horizon line is a fixed line, but not a line of fixed points. Since the image of the absolute conic, $\omega_{\infty}$, is fixed under rigid motion, there are two points that are at the intersection of the image of the absolute conic $\omega_{\infty}$ with the line, $\mathbf{l}_{h}$. They remain fixed in all images. Actually, these two fixed points 
are the image of the two circular points on the horizontal planes. These fixed image entities of the circular motion are illustrated in Figure 1.

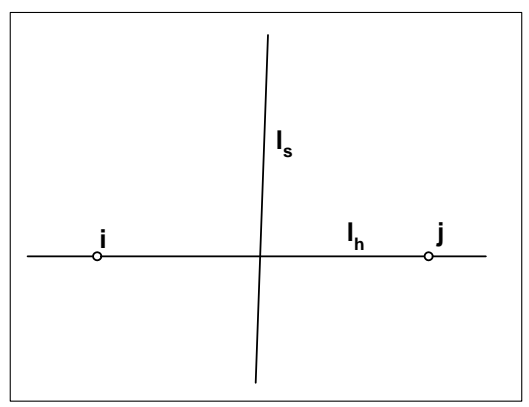

Figure 1: The fixed image entities of a circular motion: the image of fixed rotation axis $\mathbf{l}_{s}$, the image of the intersection line of all parallel planes on which the space points move.

Since the line, $\mathbf{l}_{h}$, is determined by the images of circular points, there are in total 6 d.o.f., which is enough to determine the fixed entities of the circular motion. There are 2 for each image of the two circular points and 2 for the images of the rotation axis, $\mathbf{l}_{s}$.

\section{Existing methods and redundancy analysis}

The key to any method determining the geometry of circular motion is first to determine these fixed entities from point correspondences and, then, to determine the motion parameters that are rotational angles. There are currently two existing methods:

\subsection{The three-view method using multiview geometric constraints $[8,15]$}

1. Compute one $\mathbf{F}$ from at least 7 points in 2 views to obtain the image of rotation axis, $\mathbf{l}_{s}$, and the horizon of all planes parallel to the turntable, $\mathbf{l}_{h}$, by breaking the rank-two matrix $[1,26,8]$.

2. Compute the trifocal tensor, $\mathbf{T}$, from at least one common point in 3 views to obtain the image of the circular points, $\mathbf{i}$ and $\mathbf{j}$, and the rotation angles between the three views.

This method needs at least a total of $3 \times 7=21$ points. It is evident that it is not minimal as the general three-view geometry can be solved using 6 points in 3 views $[10,6,19]$, which equals $3 \times 6=18$. 
Another observation is that the geometry of a planar motion can be solved using a 1D trifocal tensor that also requires at least 7 points in 3 views [5], which is still valid for the circular motion as the planar motion subsumes the circular motion.

\subsection{The five or $\mathrm{N}$-view method using conic fitting [11]}

Under circular motion, the trajectory of one space point is a circle and this circle projects to a conic in the image plane. Each of such conics can be fitted by the corresponding points in five images. It has been shown in [11] that two such conics are enough to recover these fixed entities of circular motion.

- Fit two conics to at least 5 corresponding points

- Compute the intersection points of the two conics to obtain the image of the circular points and, therefore, the vanishing line, $\mathbf{l}_{h}$. The rotation axis, $\mathbf{l}_{s}$ is obtained by the pole-polar relationship and the angles are computed on the conic using Laguerre's formula.

This method requires at least a total of $2 \times 5=10$ points. It naturally extends to handle any $N \geq 5$ views.

The determination of two conics is equivalent to a total of 10 points in image. They count for $2 \times 5 \times 2=20$ d.o.f. as each point has 2 d.o.f. in an image. After subtracting the 6 d.o.f. fixed entities of the circular motion and the 6 d.o.f. of the two points in space, the 8 d.o.f. remaining are the rotation angles associated with all images involved. As the 10 tracked points can spread at most over 2 different groups of 5 images, the 8 d.o.f. remaining can be counted for the 4 rotation angles of each group of 5 images. The case of two conics is therefore minimal for this particular case of 2 groups of 10 images. But it is redundant in any other case. For instance, for a common case where the two points are tracked over the same 5 images, it is highly redundant as only 4 parameters need to be determined from the 8 given ones.

There is also a mixture of one conic and one fundamental matrix method presented in [11].

\section{The minimal method using 2 points in 4 images}

Motivated by the non-minimality of existing methods $[8,11]$ in terms of the total number of points used to determine the geometry, we describe a minimal solution using only 2 points in 4 images 
totaling only 8 points in this section. Analysis and proofs of the results are given in the next section.

1. Given a minimum of 2 distinct points tracked over 4 images, $\mathbf{a}_{i}$ and $\mathbf{b}_{i}$, for $i=1 \ldots 4$.

2. Compute a $3 \times 3$ plane homography $\mathbf{H}$ such that

$$
\lambda \mathbf{b}_{i}=\mathbf{H a} \mathbf{a}_{i}, \text { for } i=1 \ldots 4
$$

This can be easily solved using the standard SVD method with normalized image coordinates [10].

3. Compute the eigenvalues and eigenvectors of this homography matrix, $\mathbf{H}$. The eigenvectors corresponding to the pair of complex conjugate eigenvalues are the image of the pair of circular points $\mathbf{i}$ and $\mathbf{j}$.

As $\mathbf{H}$ is a $3 \times 3$ matrix, this is equivalent to solving a cubic equation with real coefficients. It may have either three real or one real and a pair of complex conjugate eigenvalues. By the circular motion constraint, it is impossible to have three real eigenvalues. Two of the eigenvalues have to be a pair of conjugate complex numbers.

4. Determine the horizon line as the line going through the images of the circular points:

$$
\mathbf{l}_{h}=\mathbf{i} \times \mathbf{j}
$$

5. Fit conics $\mathbf{C}_{a}$ and $\mathbf{C}_{b}$ respectively to the 4 tracked points $\mathbf{a}_{i}$ and $\mathbf{b}_{i}$, and the images of the circular points, $\mathbf{i}$ and $\mathbf{j}$.

The key here is that, under circular motion, the trajectory of one space point is a circle and this circle projects to a conic in the image plane. Each tracked point has a different conic locus, but all conics must go through the images of the circular points.

6. Extract rotation axis, $\mathbf{l}_{s}$ and the rotation angles from the conics.

This is the same as described in [11]. We briefly summarize as follows:

The pole of each conic with respect to the the horizon line, $\mathbf{l}_{h}$, is given by

$$
\mathbf{o}_{a}=\mathbf{C}_{a}^{-1} \mathbf{l}_{h}, \text { and } \mathbf{o}_{b}=\mathbf{C}_{b}^{-1} \mathbf{l}_{h}
$$


They are the images of the center of the two space circles and lie on the image of the rotation axis, $\mathbf{l}_{s}$. The rotation axis can be calculated as $\mathbf{l}_{s}=\mathbf{o}_{a} \times \mathbf{o}_{b}$.

The rotation angle between the images, $i$ and $j$, is calculated by using Laguerre's formula [22]:

$$
\theta_{i j}=\frac{1}{2 i} \ln \left(\left\{\mathbf{o}_{a} \times \mathbf{a}_{i}, \mathbf{o}_{a} \times \mathbf{a}_{j} ; \mathbf{o}_{a} \times \mathbf{i}, \mathbf{o}_{a} \times \mathbf{j}\right\}\right) .
$$

It is interesting to note that solving a cubic equation is reminiscent of similar situations in selfcalibration using both a special trifocal tensor [1] and a 1D trifocal tensor [5]. The trifocal tensor needs many more points to be determined, 6 points in 3 images, but here only 2 points in 4 images.

\section{Construction and analysis of the homography $\mathbf{H}$}

In this section, we will first prove that the tracked image points, $\mathbf{a}_{i}$ and $\mathbf{b}_{i}$, in the same images are related by a homography. The proof is a geometric construction of this homography. Then, this homography will be analyzed to prove the results used in the previous section.

\subsection{Construction of the homography $H$}

In space, consider two points, $\mathbf{A}$ and $\mathbf{B}$, circularly moving around a fixed axis in different planes, $\pi_{A}$ and $\pi_{B}$. The trajectory of points $\mathbf{A}$ and $\mathbf{B}$ are $\mathbf{A}_{i}$ and $\mathbf{B}_{i}$ at each different position.

Now, look at the relationship between $\mathbf{A}_{i}$ and $\mathbf{B}_{i}$. The very important fact is that $\mathbf{A}_{i}$ and $\mathbf{B}_{i}$ have the same relative angles between them by the very definition of the circular motion, i.e., the angle between $\mathbf{A}_{i}$ and $\mathbf{A}_{j}$ on its circular trajectory is always equal to that between $\mathbf{B}_{i}$ and $\mathbf{B}_{j}$ on its own cricular trajectory. The trajectories of the points $\mathbf{A}_{i}$ and $\mathbf{B}_{i}$ are two circles in different planes, these two circles uniquely define a cone or a cylinder (if $\mathbf{A}_{i}$ and $\mathbf{B}_{i}$ happen to have the same radius for their circular trajectories). The cone vertex is always on the fixed rotation axis, but it might be located on the same side of the circles as shown in Figure 3, or between the two circles, or at infinity if the two circles have the same radii. A point-to-point correspondence can be established between the points of the two circles by drawing lines from the vertex. The line connecting vertex $\mathbf{V}$ and point $\mathbf{A}_{i}$ yields the corresponding point $\mathbf{A}_{i}^{\prime}$. The angle between $\mathbf{A}_{i}^{\prime}$ and $\mathbf{A}_{j}^{\prime}$ is preserved by this construction, the same as that of $\mathbf{A}_{i}$ and $\mathbf{A}_{j}$. And the angle between $\mathbf{A}_{i}$ and $\mathbf{A}_{j}$ is the same as that of $\mathbf{B}_{i}$ and $\mathbf{B}_{j}$, so that there 
exisits a rotation around the circle's center with an angle $\theta$ such that

$$
\mathbf{B}_{i}=\mathbf{R}(\theta) \mathbf{A}_{i}^{\prime}
$$

where the angle $\theta$ is the angle between the space points $\mathbf{A}$ and $\mathbf{B}$ on the object rotating around the rotation axis.

(a)

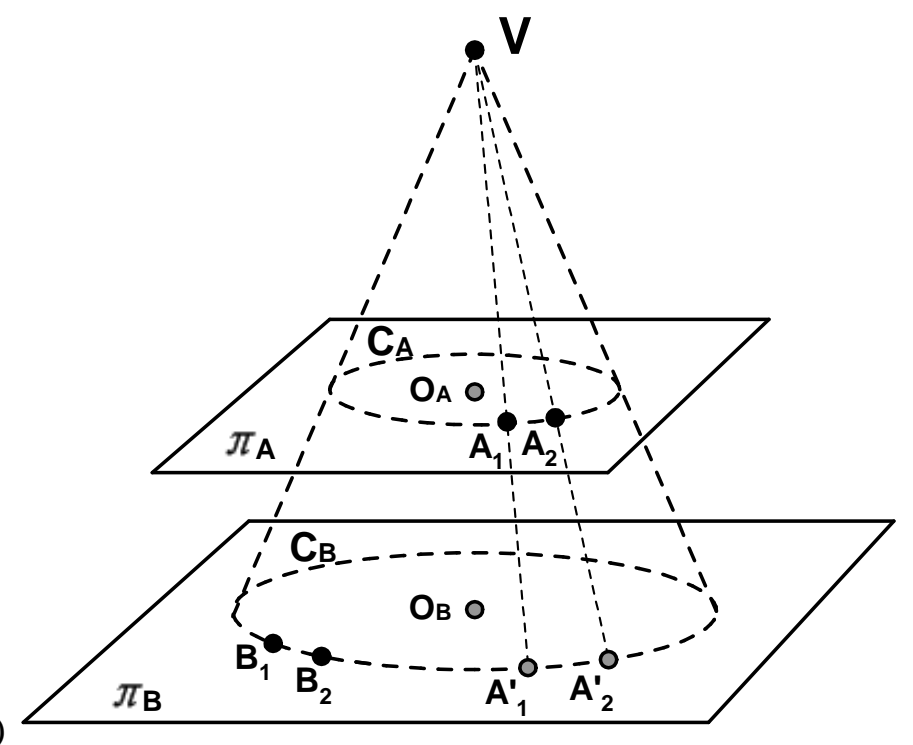

(b)

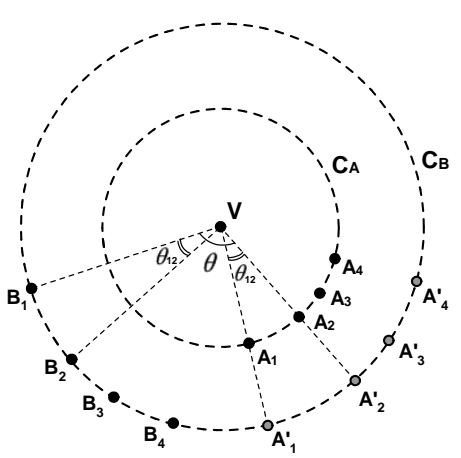

Figure 2: The geometric configuration in 3D space. (a) The space points $\mathbf{A}$ and $\mathbf{B}$ are circularly moving around the fixed rotation axis on two different planes. Two circles determine a cone. A correspondence is established between the points on the two circles by drawing lines from the cone vertex. (b) A top view along the rotation axis of the geometry. The angle between $\mathbf{A}_{i}$ and $\mathbf{A}_{j}$ is the same as between $\mathbf{B}_{i}$ and $\mathbf{B}_{j}$ which is the rotation angle at the different positions. The angle $\theta$ is the angle between the two space points around the rotation axis.

Now let us come back to the image space. Consider the image by a projective camera of this geometric configuration in space. The transformation between the image plane and the plane, $\pi_{A}$, on 
which A moves is a planar projective transformation, i.e., a planar homography, $\mathbf{H}_{A}$,

$$
\lambda \mathbf{a}_{i}=\mathbf{H}_{A} \mathbf{A}_{i}
$$

The same is for the point $\mathbf{B}$ and its image points, $\mathbf{b}_{i}, \lambda \mathbf{b}_{i}=\mathbf{H}_{B} \mathbf{B}_{i}$. The circular trajectories are projected onto conics. The rotation axis being fixed in space is projected onto the fixed image line, $\mathbf{l}_{s}$, and the intersection line of the parallel planes is the fixed vanishing line $\mathbf{l}_{h}$. As all lines in space connecting $\mathbf{A}_{i}$ and $\mathbf{A}_{i}^{\prime}$ lying on two planes are concurrent at the vertex, $\mathbf{V}$, which naturally results in a planar homology, $\mathbf{G}$ for the image points $\mathbf{a}_{i}$ and $\mathbf{a}_{i}^{\prime}$, i.e.,

$$
\lambda \mathbf{a}_{i}^{\prime}=\mathbf{G} \mathbf{a}_{i}
$$

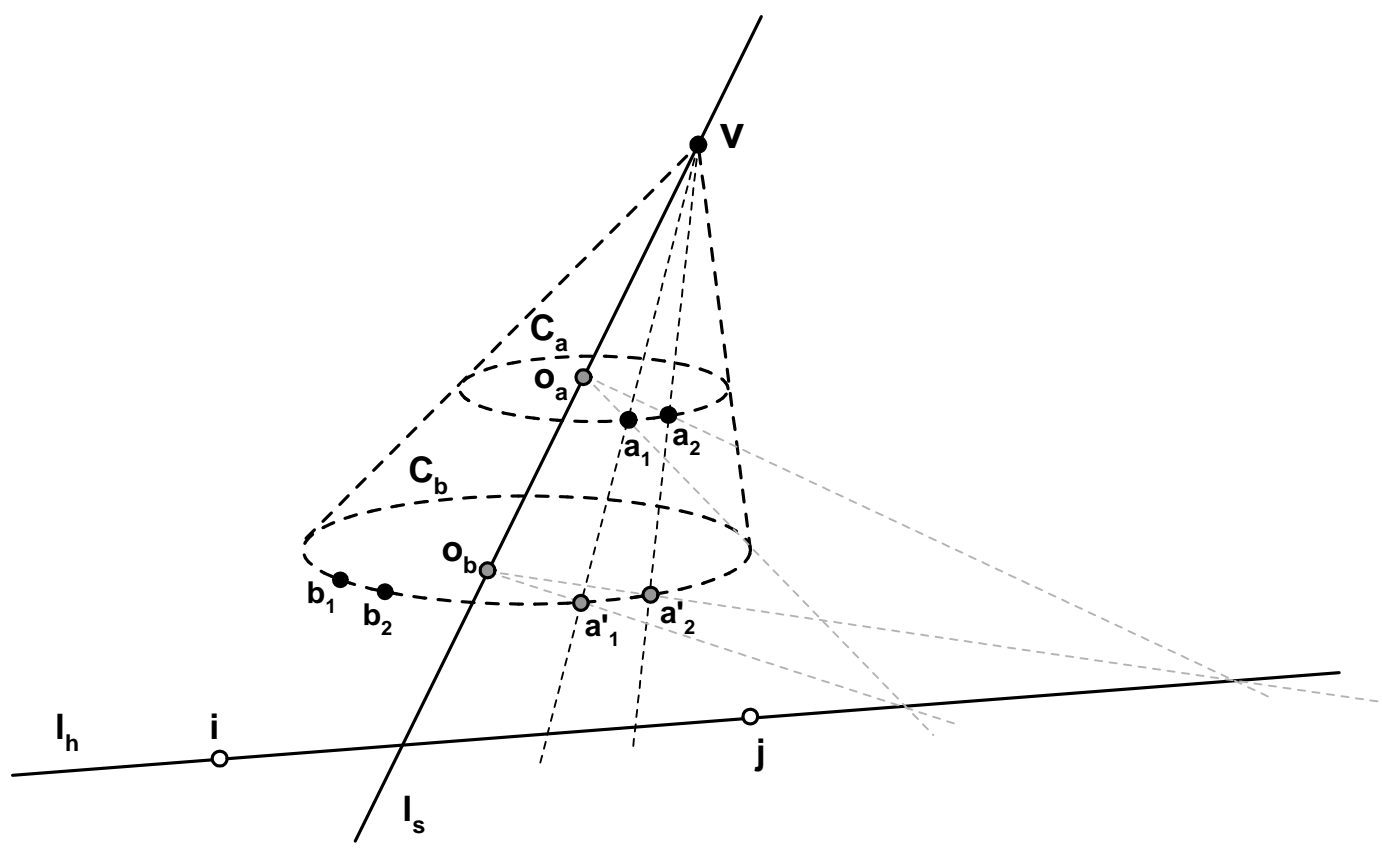

Figure 3: The geometric configuration in the image plane. The image points $\mathbf{a}_{i}$ and $\mathbf{a}_{i}^{\prime}$ are related by a homology. The fixed line of the homology is the horizon line $\mathbf{l}_{h}$, and the fixed point is the image of the cone vertex. $\mathbf{l}_{s}$ is the image of the rotation axis.

The homology [22] arises in other vision geometry in [25] and is also discussed in [10]. A planar homology is a special plane projective transformation that has a line of fixed points and a fixed point not on the line. Algebraically, it has a repeated eigenvalue, and, therefore, algebraic multiplicity of 2. Its algebraic multiplicity is equal to its geometric multiplicity $[22,10]$. It also transforms a conic into another one. 
It is clear that the fixed line of the homology, $\mathbf{G}$, is the horizon line, $\mathbf{l}_{h}$, and the fixed point is the vertex, v. This homology, G, can therefore be parameterized [10] as

$$
\mathbf{G}=\mathbf{I}+(\mu-1) \frac{\mathbf{v} \mathbf{l}_{h}^{T}}{\mathbf{v}^{T} \mathbf{l}_{h}}
$$

where $\mathbf{I}$ is the identity matrix and $\mu$ is the characteristic invariant of the planar homology. The geometric interpretation of the characteristic $\mu$ is the cross ratio of the vertex, $\mathbf{v}$, any corresponding homological points, and the intersection point of the connected line of the corresponding points with $\mathbf{l}_{h}$.

Finally, using $\mathbf{a}_{i}^{\prime}=\mathbf{H}_{B} \mathbf{A}_{i}^{\prime}$, the homography for $\mathbf{b}_{i}=\mathbf{H} \mathbf{a}_{i}$ is completely and explicitly chained as follows:

$$
\mathbf{H}=\mathbf{H}_{B} \mathbf{R}(\theta) \mathbf{H}_{B}^{-1} \mathbf{G}
$$

The 8 d.o.f. of the planar homography, $\mathbf{H}$, are naturally distributed as follows. The planar homology, G, has 5 d.o.f., where the vertex, $\mathbf{v}$, has 2 d.o.f., the axis, $\mathbf{l}_{h}$, has 2 d.o.f. and the characteristic invariant $\mu$ has 1 d.o.f. The homography matrix, $\mathbf{H}_{B}$, is between the image plane and the space plane on which the point $\mathbf{B}$ moves. It can be parameterized by the images of two circular points $[14,11]$ having 4 d.o.f.. However, since the fixed line, $\mathbf{l}_{h}$, has been counted in the homology, $\mathbf{G}$, the homography, $\mathbf{H}_{B}$, counts only for 2 addtional d.o.f.. The rotation matrix, $\mathbf{R}(\theta)$, counts for 1 d.o.f. In a different classification, the 8 degrees of freedom of the homography contains the 6 motion parameters (the image of the circular points (4) and the vertex $\mathbf{v}(2)$ ) and 2 structure parameters ( $\mu$ for the relative position of the planes $\pi_{A}$ and $\pi_{B}$ and $\theta$ for the angle between the points $\mathbf{A}$ and $\mathbf{B}$ ).

\subsection{Eigenvectors of $\mathbf{H}$}

Now, we prove that the image of the circular points are the eigenvectors of the homography, $\mathbf{H}$, associated with the pair of complex conjugate eigenvalues.

Special case We first consider the special case when $\mu$ is equal to 1 , but $\theta$ is different from 0 or $\pi$. This corresponds to the configuration of the two circles lying on the same plane and the homology 
therefore degenerates into the identity matrix. The homography becomes

$$
\mathbf{H}=\mathbf{H}_{B} \mathbf{R}(\theta) \mathbf{H}_{B}^{-1}
$$

The eigenvalues of $\mathbf{R}$ are 1 and a pair of complex conjugates, $e^{ \pm i \theta}$. The eigenvectors of $\mathbf{R}$ corresponding to the pair of conjugate complex eigenvalues are the circular points $(1, \pm i, 0)^{T}$.

The homography, $\mathbf{H}$ is a similar matrix to $\mathbf{R}$. It keeps the eigenvalues but transforms the eigenvectors into the images of the circular points, $\mathbf{H}_{B}(1, \pm i, 0)^{T}$.

General case The general case is when $\theta$ is not equal to 0 or $\pi$, and $\mu$ is different from 1 . The images of the circular points are $\mathbf{H}_{B}(1, \pm i, 0)^{T}$ and they are lying on the line, $\mathbf{l}_{h}$, which is a fixed line of the homology by its definition. The homology leaves the line $\mathbf{l}_{h}$ invariant. Therefore, the images of the circular points on this line are invariant. This amounts to saying that the images of the circular points are invariant vectors of the homography. This proves that the eigenvectors corresponding to the pair of complex conjugate eigenvalues are generally the images of the circular points.

Degeneracy The homography, $\mathbf{H}$, degenerates to the homology, $\mathbf{G}$, when $\theta=0$. When $\theta=\pi$, it is equivalent to the case where the cone vertex is located in between two circles, and $\theta=0$. Then, $\mathbf{H}$ degenerates to a homology, $\mathbf{G}$ :

$$
\mathbf{H}=\mathbf{G}=\mathbf{I}+(\mu-1) \frac{\mathbf{v} \mathbf{h}_{h}^{T}}{\mathbf{v}^{T} \mathbf{l}_{h}} .
$$

Algebraically a homology has two equal and one distinct eigenvalues [22, 10]. Clearly, the real eigenvalue of 1 is repeated twice. As the corresponding eigenvectors are points on $\mathbf{I}_{h}$, we can not distinguish the value of the images of the circular points when these 8 points satisfy a homology.

This degenerate case can be easily detected in practice by its geometric construction: the four lines connecting the corresponding points meet at the vertex point.

\subsection{Method minimality}

Our new method uses as few as 2 points tracked in 4 images to recover the full geometry of circular motion. We prove that indeed 2 points is a minimum number for 4 images by counting the degrees of freedom of the system. 
Each point has 2 d.o.f. in an image and 3 d.o.f. in 3D space. The given $8=2 \times 4$ image points have a total of $2 \times 4 \times 2=16$ d.o.f., which counts for a total of 10 d.o.f. for the 4 camera motion parameters and $6=2 \times 3$ for the 2 reconstructed points in space. The 10 d.o.f. for the 4 camera motion parameters are the images of the circular points $(2 \times 2$ d.o.f. $)$, the image of the rotation axis ( 2 d.o.f.), the characteristic invariant $\mu$ (1 d.o.f.) and the three rotation angles related to these four images ( 3 d.o.f.). The 2 points in 4 images are therefore minimal.

\section{Robust optimization using RANSAC}

The minimal solution can be easily plugged into an optimization procedure in which we can remove the tracking outliers. In this section, we describe a complete optimization procedure using RANSAC for a modeling system.

1. Arrange the data set $S$.

The tracking data are structured into a quadruplet of the image coordinates $\left\{\mathbf{x}_{1}, \mathbf{x}_{2}, \mathbf{x}_{3}, \mathbf{x}_{4}\right\}$ for each point tracked in 4 images. Note that it is unnecessary to assume that the 4 images are consecutive.

2. Compute the fixed image entities using the new method of 2 points in 4 images developed in this paper.

Randomly select 2 points in 4 images from the data set, $S$. Check whether the configuration of these 2 sets of 4 points degenerates into a homological relationship. The test is done by checking the numerical rank of the matrix stacking all these points together using SVD.

If these points are not related by a homography, compute a homography. Then, extract the images of the circular points, $\mathbf{i}$ and $\mathbf{j}$, the image of the rotation axis, $\mathbf{l}_{s}$, and the horizon line, $\mathbf{l}_{h}$, from the homography.

3. Compute an evaluation criterion for each tracked point in 4 images from the data set, $S$.

First, a conic is fitted to the 4 points plus 2 points that are the image of the circular points by SVD. Then, an approximate point-conic distance is defined to be $[2,20,10]$ 


$$
d(\mathbf{x}, \mathbf{C})^{2}=\sum_{i=1}^{4} \frac{\left(\mathbf{x}_{i}^{T} \mathbf{C} \mathbf{x}_{i}\right)^{2}}{4\left(\left(\mathbf{C} \mathbf{x}_{i}\right)_{1}^{2}+\left(\mathbf{C} \mathbf{x}_{i}\right)_{2}^{2}\right)}
$$

where $(\mathbf{C x})_{m}$ denotes the $m$-th component of the three-vector $\mathbf{C x}$.

In addition to the fact that the image of the circular points goes through the conic, there exists a constraint, $\left(\mathbf{C}^{-1} \mathbf{l}_{h}\right)^{T} \mathbf{l}_{s}=0$. To take this constraint into account, we compute the following point-line distance:

$$
d\left(\mathbf{l}_{s}, \mathbf{o}\right)^{2}=\frac{\left(\mathbf{l}_{s}^{T} \mathbf{o}\right)^{2}}{\left(\mathbf{l}_{s}\right)_{1}^{2}+\left(\mathbf{l}_{s}\right)_{2}^{2}},
$$

where $\mathbf{o}$ is the normalized pole of the $\mathbf{l}_{h}$ with respect to the fitted conic $\mathbf{C}$, and $\left(\mathbf{l}_{s}\right)_{m}$ is the $m$-th component of the 3 -vector $\mathbf{l}_{s}$.

We then define the following criterion $\mathcal{C}_{\mathbf{X}}$ for each tracked point in 4 images w.r.t. the current fixed image entities as

$$
\mathcal{C}_{\mathbf{X}}=d(\mathbf{x}, \mathbf{C})^{2}+\lambda d\left(\mathbf{l}_{s}, \mathbf{o}\right)^{2}
$$

where $\lambda$ is Lagrange multiplier.

4. Compute the percentage of inliers consistent with the selected 2 points in 4 images using the evaluation criterion $\mathcal{C}_{\mathbf{X}}$ with a threshold $T$.

5. Repeat $N$ times from step 2 to 4 for choosing the 2 points in 4 images with the largest number of inliers. $N$ and $T$ are determined by the proportion of inliers [10].

6. Compute an optimal solution using all the inliers. The maximum likelihood estimate is obtained by minimizing $\mathcal{C}=\sum \mathcal{C}_{\mathbf{X}}$. This nonlinear minimization problem is solved using the LevenbergMarquart equation from the initial estimates obtained from the minimal data set of 2 points in 4 images.

\section{Experiments}

The new method of computing circular motion geometry from a minimum of 2 points in 4 images is implemented and tested. 


\subsection{Chessboard experiment}

We first set up an experiment to verify the accuracy of the results using the minimal data. Two chessboards are put on a rotary table. The four images of the chessboards captured at different positions are shown in Figure 4. The lines are detected for the chessboards using an existing program. Then, corresponding point features are taken to be all the intersection points of these lines thanks to the regularity of the chessboard pattern. There are 70 points in 4 images, and more than 2000 sets of minimal data can be obtained for the computation.
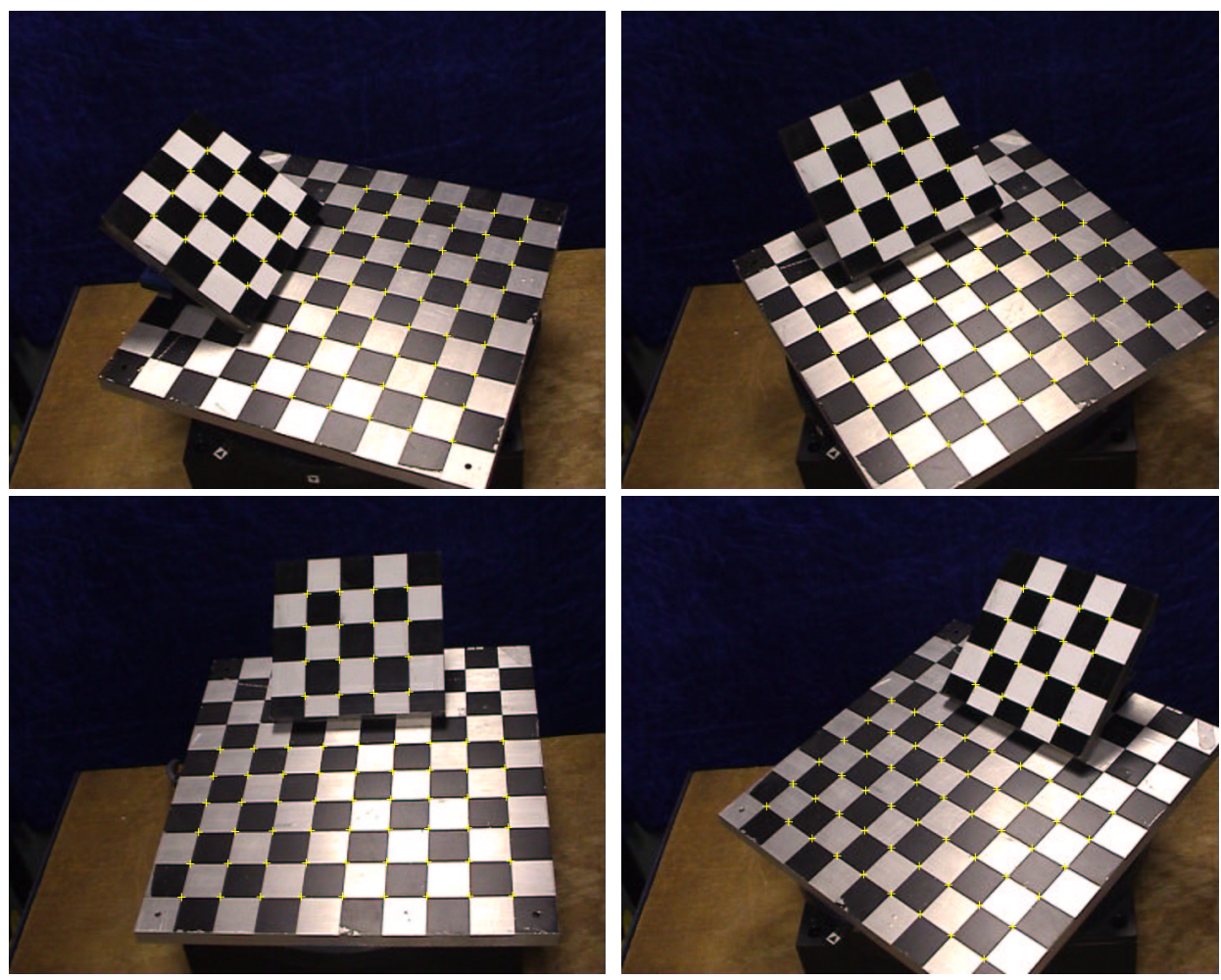

Figure 4: The four images of the chessboards captured at different positions and the corresponding point features computed as the intersection points of the lines detected for these images.

We may first examine the distribution of the computed coordinates of the image of circular points $(a \pm b i, c \pm d i, 1)^{T}$. A histogram from all sets of data is drawn in Figure 5 for each component, $a$, $b, c$ and $d$. The distribution is without any surprise close to a Gaussian. Most of theses are very good results, which means that the minimal data computation is useful in practice to bootstrap either a robust method or an optimization method. There are also some computed values far away from the peak of the curve. In fact, many of those configurations are close to the degenerate cases of 
the homological points as discussed in Section 6. This includes two cases: the first is when the intersection of the lines connecting points on the different conics is close to the image of the rotation axis; the second is when the space point is close to the rotation axis.
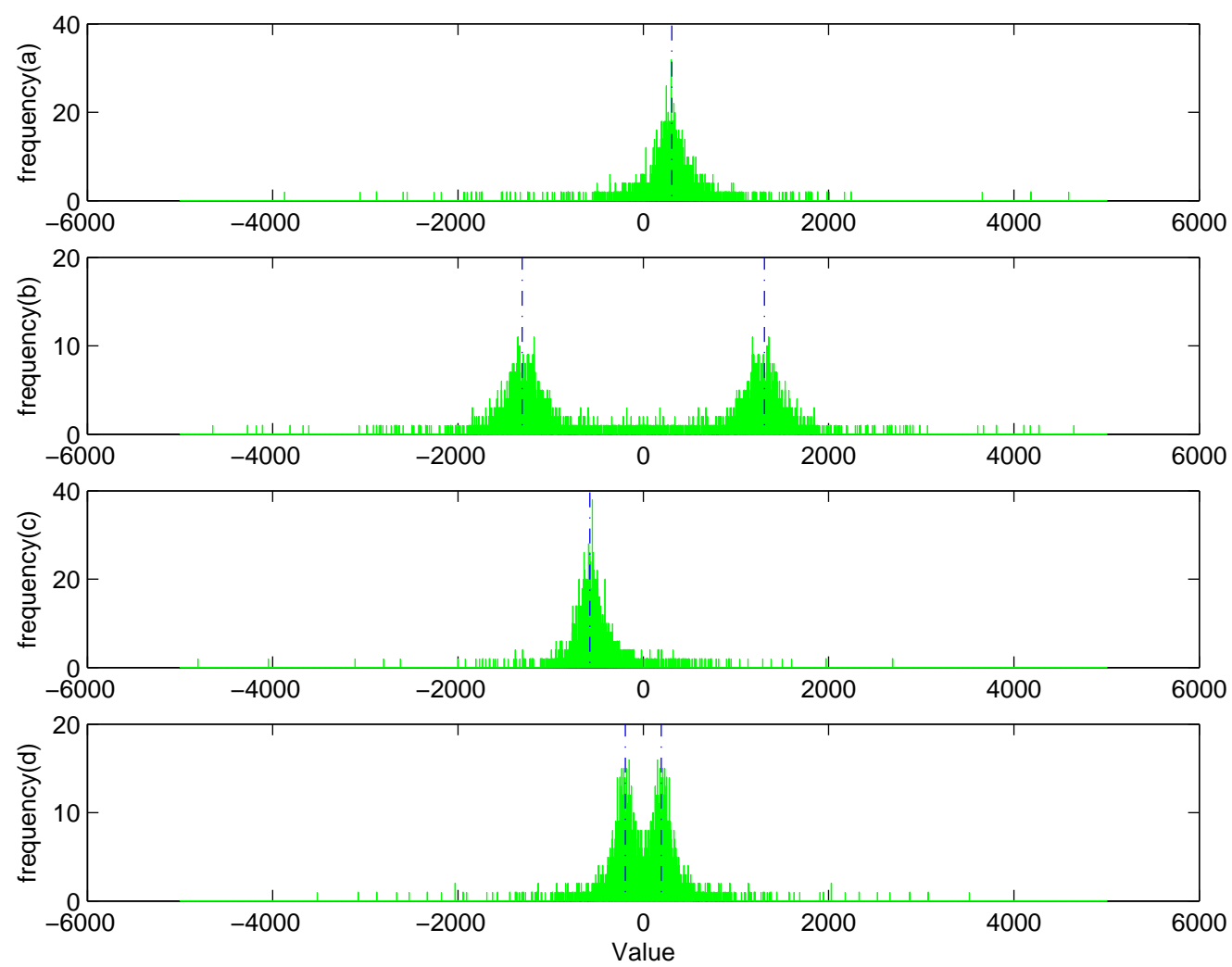

Figure 5: The histograms of $a, b, c$, and $d$, which are coordinates of the image of circular points $(a \pm b i, c \pm d i, 1)^{T}$. The dashed lines are the optimized solutions automatically computed.

Figure 6 shows the recovered horizon line, $\mathbf{l}_{h}$, and the image of rotation axis, $\mathbf{l}_{s}$, from the data sets giving inlier results for $a, b, c$ and $d$. We should also avoid the case where the two points are close to coplanar for a stable computation of $\mathbf{l}_{s}$.

The data set automatically selected by the RANSAC-driven optimization is shown in Figure 7. The points marked as squares are the minimal data set selected by RANSAC for subsequent optimization. The gray points are inliers and the dark colored points are outliers. Inlier gray colored points are used to fit conics. The relative angles between these four images are computed as 37.108, 37.144, and 36.579 degrees. Optimized solutions are also shown in Figure 5 and Figure 6 with dashed lines. 


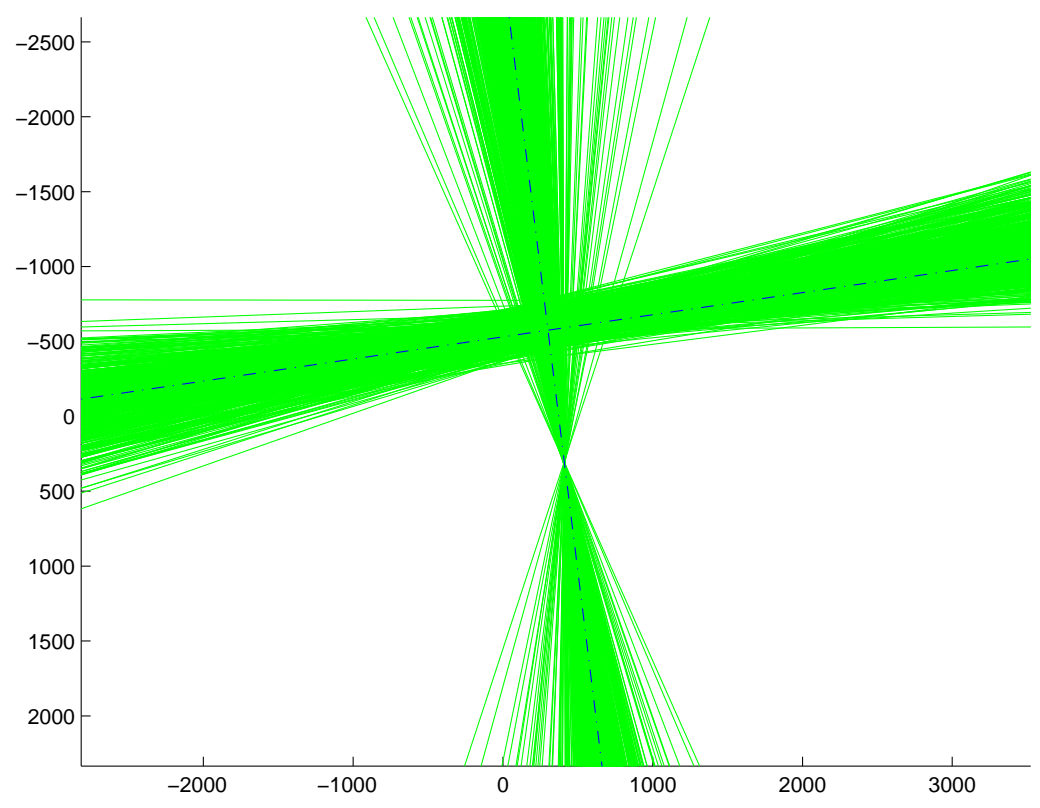

Figure 6: Results of recovered horizon line $\mathbf{l}_{h}$ and the image of rotation axis $\mathbf{l}_{s}$ with different minimal data set. The dashed lines are the optimized solutions.

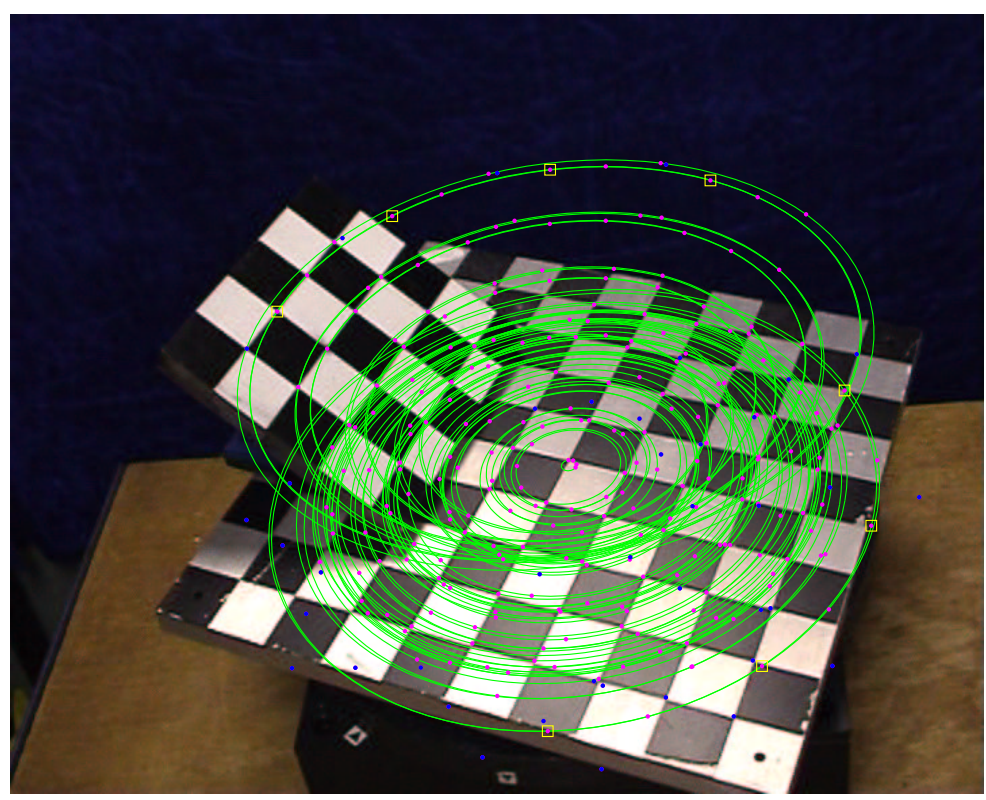

Figure 7: The results computed by RANSAC and optimization. The minimal data set selected by RANSAC are marked in squares. The gray colored points are inliers, and the dark colored points are outliers. These gray colored points are fitted to corresponding conic loci. 


\subsection{Proceedings experiment}

We then set up an experiment to verify the independency of the computation of the invariant geometric entities upon the selection of images. The six images of the ICCV proceedings shown in Figure 8 are captured at different positions. The points of interest are detected for each image and the corresponding points are computed in these six images. The computation is not restricted to any particular set of four images. Any set of four images can contribute to the computation as long as the set of four images has sufficient tracked points. The RANSAC-driven method automatically selects a minimal data set in the images 1,2, 4, and 5 shown in square marks in Figure 8.

Figure 9 shows the inlier and outlier points validated in one of the original images that contains the selected minimal data. The fitted conic trajectories in the image allow to check the accuracy of the estimated geometry for the inlier points.

Figure 10 shows the inlier and outlier points in a different image that does not contain any initial minimal data. The points in this image are used to compute the final optimized solutions. The computed angles between these six images are 29.868, 36.059, 22.985, 30.830, and 35.598 degrees.

The images of circular points are obtained as $(8.4510 e 2 \pm 4.2177 e 3 i,-1.2354 e 3 \mp 8.1546 e 2,1)^{T}$. The image of rotation axis is located at $\mathbf{l}_{s}=(-1.0927 e-3,2.8665 e-4,1)^{T}$.

\subsection{Dinosaur experiment}

We also tested our implementation on the popular dinosaur sequence from University of Hannover (available from University of Oxford [9]). The sequence contains 36 views from a turntable with a constant angular motion of 10 degrees. The angular accuracy is about 0.05 degrees [18]. Figure 11 shows one of the images of the dinosaur sequence and some tracked points along all images. The selected corresponding points are those that have been tracked over at least 4 images and longer camera distances.

The data set selected by RANSAC marked as squares is shown in Figure 12 in which the gray colored points are inliers and the dark colored points are outliers. The computed results of the images of circular points are $(266.04 \pm 3156.3 i,-1151.9 \mp 88.111)^{T}$.

The computed images of $\mathbf{l}_{h}$ and $\mathbf{l}_{s}$ are shown in Figure 13. The rotation angles calculated from the inlier points are shown in Figure 14. 

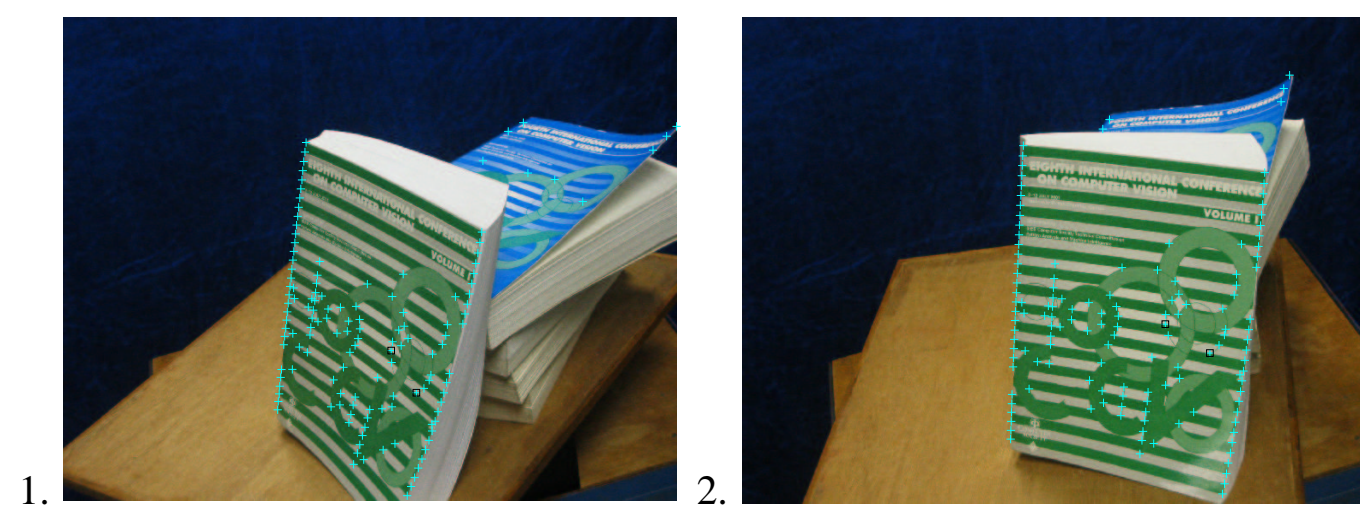

3.
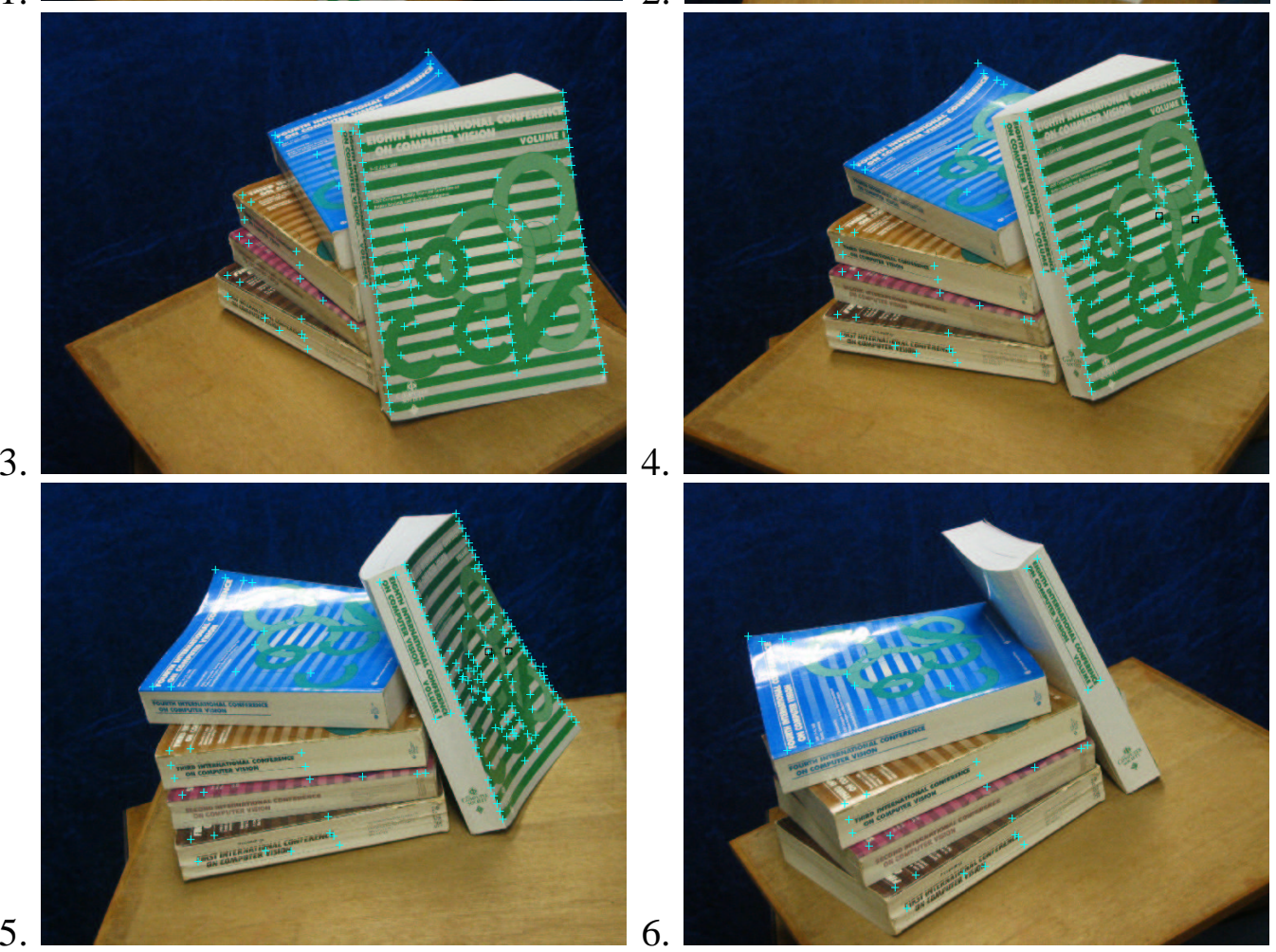

Figure 8: The six images of the proceedings captured at different positions and the corresponding points of interest. The square marks in the images $1,2,4$, and 5 are the minimal data selected by RANSAC. 


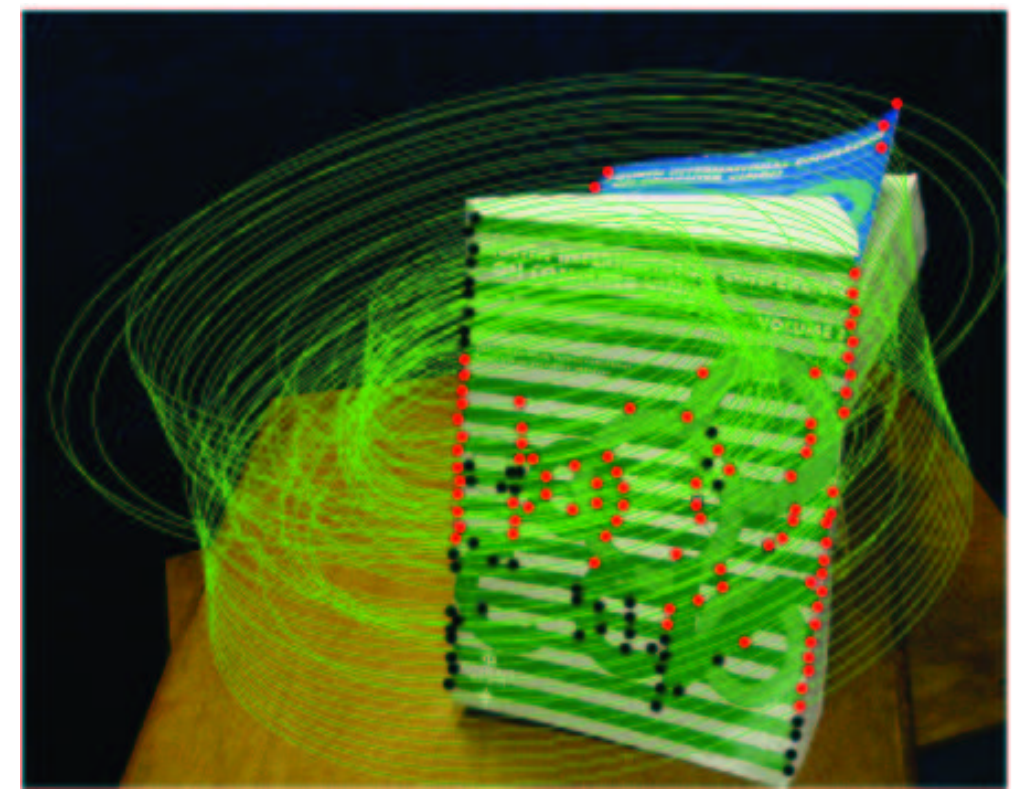

Figure 9: Inlier (gray mark) and outlier (dark mark) points validated by the computed geometry and the fitted conics for inlier points.

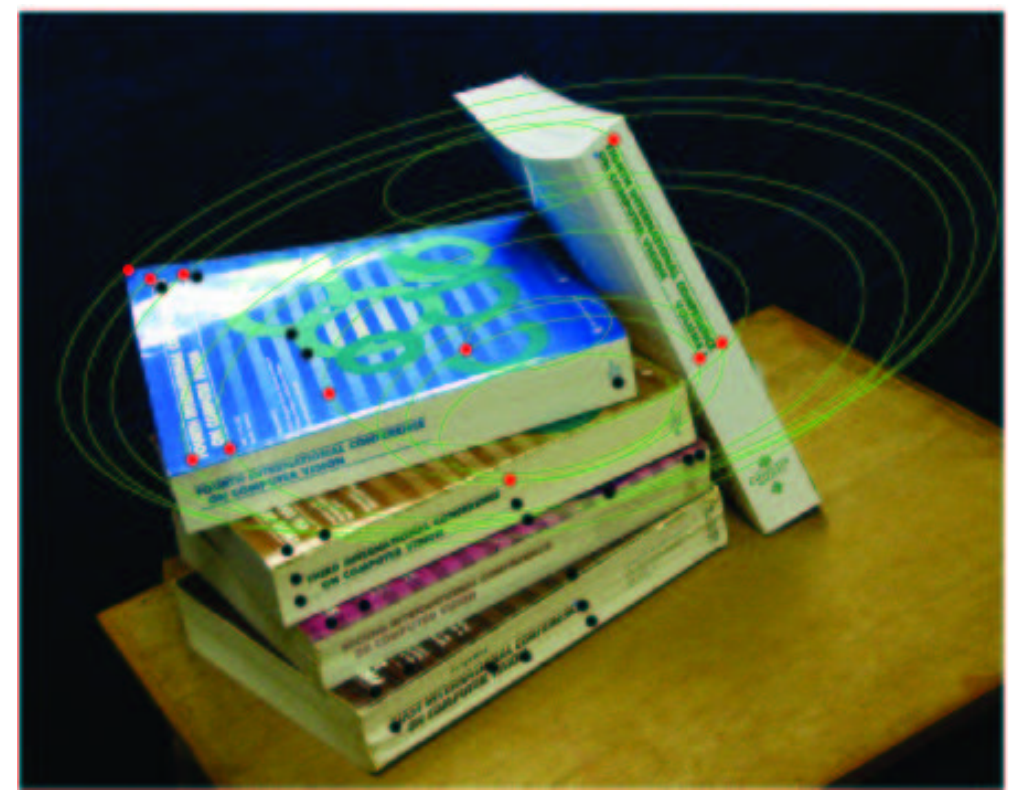

Figure 10: Inlier (gray mark) and outlier (dark mark) points validated by the computed geometry and the fitted conics for inlier points. This image is not used for the geometry computation using the minimal data, but is used for the final optimization. 


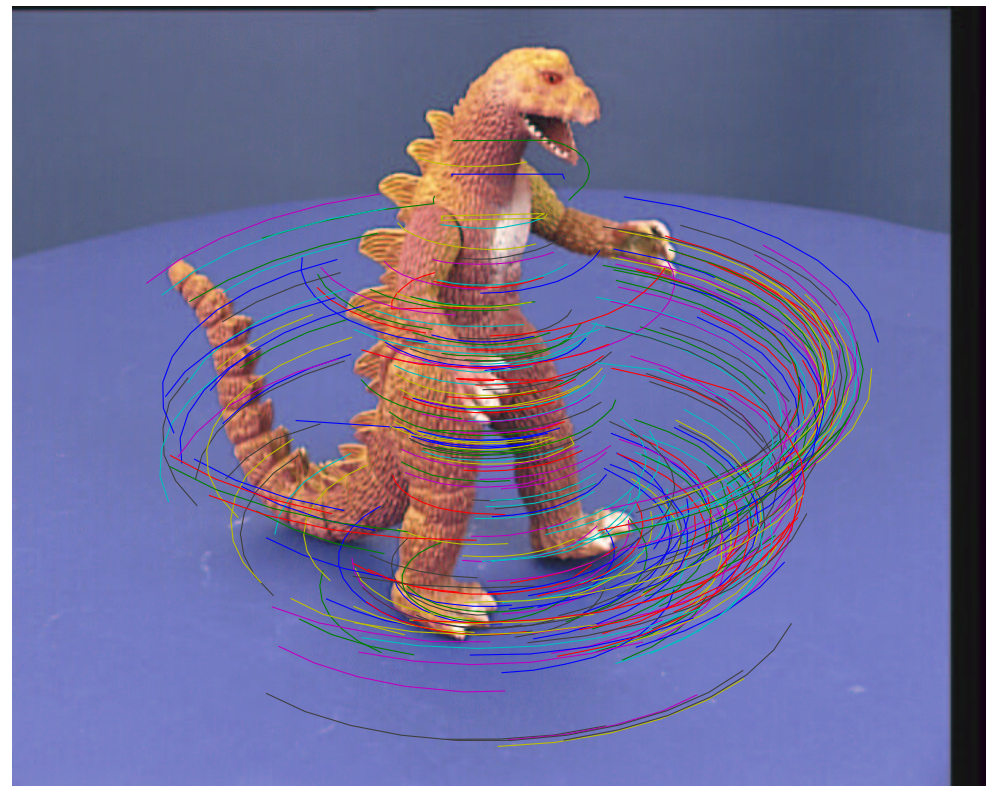

Figure 11: One of dinosaur sequence and selected corresponding points.

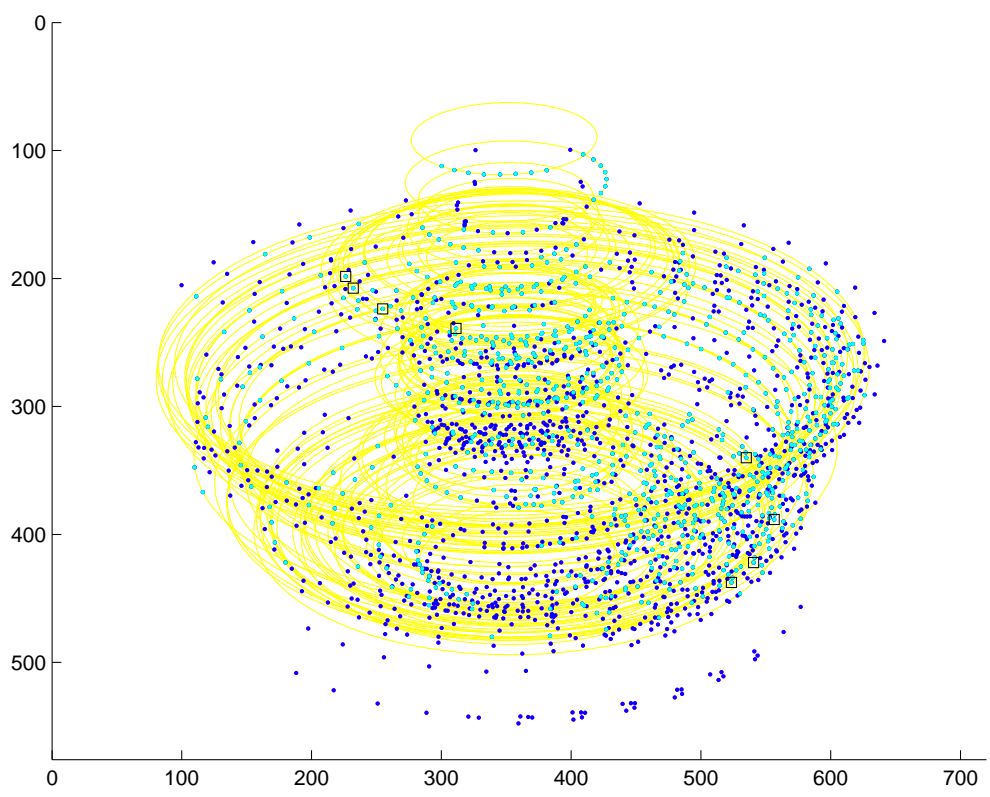

Figure 12: The results computed by RANSAC and optimization. The minimal data set selected by RANSAC are marked in squares. The gray colored points are inliers, and the dark colored points are outliers. These gray colored points are fitted to corresponding conic loci. 


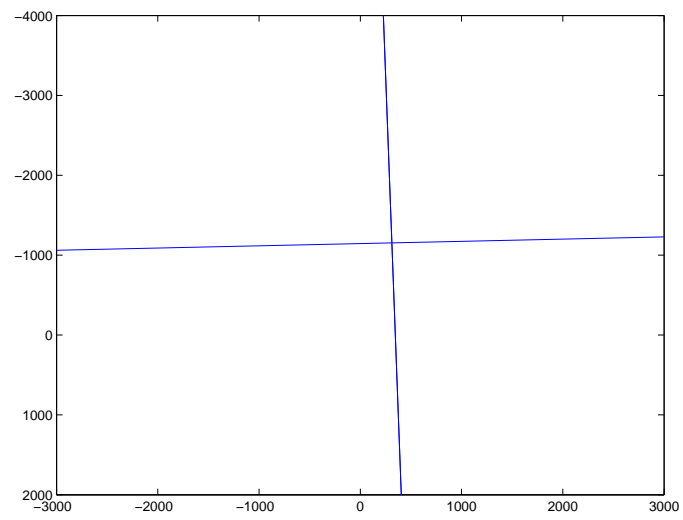

Figure 13: The computed images of rotation axis $\mathbf{l}_{s}$ and the horizon line $\mathbf{l}_{h}$.

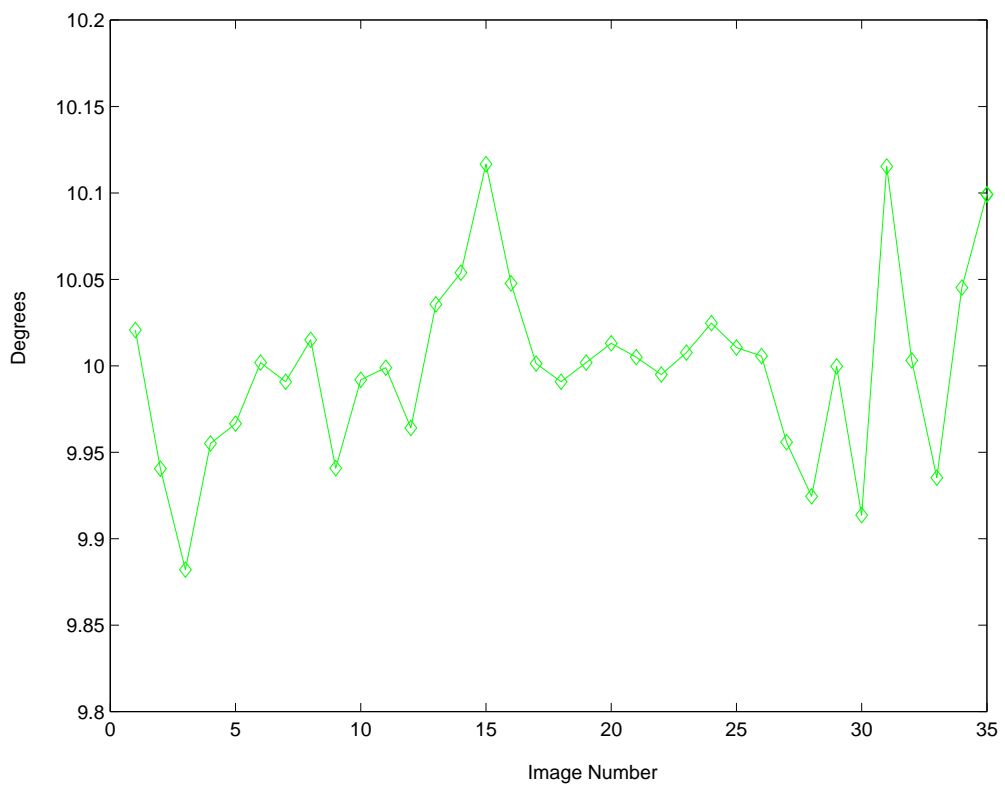

Figure 14: The computed rotation angles for the whole sequence of 36 views. 
If we fix the aspect ratio of the camera and assume a point on the image of rotation axis as a vanishing point $[8,11]$, the visual hull of the dinosaur can be computed $[4,18]$. The excellence of the computed visual hull as shown in Figure 15 depends on the accuracy of the geometry estimation.
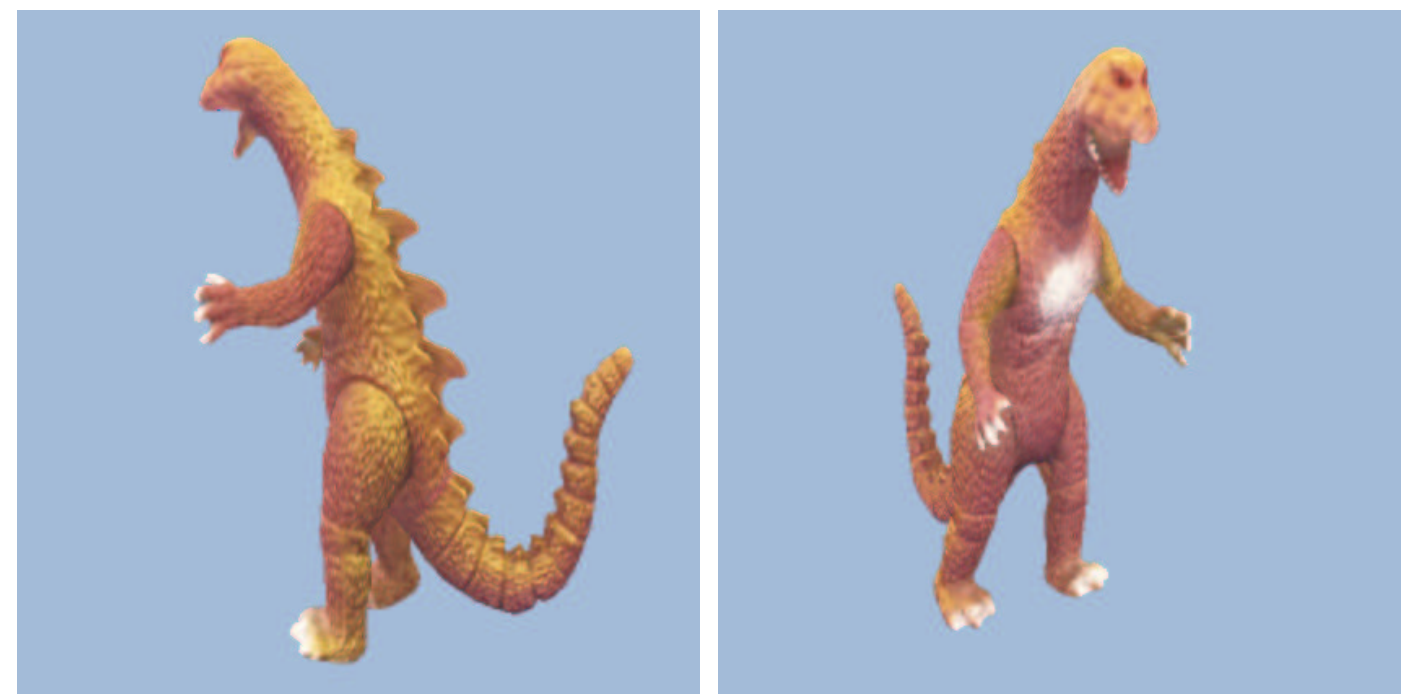

Figure 15: Two views of the 3D reconstruction of dinosaur from silhouettes.

\section{Conclusion}

This paper presented a new and simple algorithm for computing the geometry of circular motion or single axis motion using a minimal data set of two points in four images. This method is minimal compared with existing methods that use either multi-view constraints and conic fitting. The algorithm is also remarkably simple as we only need to compute a three by three homography, and then extract its eigenvalues and eigenvectors. The experiments on real sequences demonstrate the usability of this minimal solution. We also developed a RANSAC-driven optimization using initial values provided by this minimal data solution.

\section{Acknowledgements}

This work is partially supported by the Hong Kong RGC Grant CUHK4378/02E and HKUST6188/02E. 


\section{References}

[1] M. Armstrong, A. Zisserman, and R. Hartley: Self-Calibration from Image Triplets, In Proc. European Conference on Computer Vision, pp. 3-16, 1996.

[2] F. Bookstein: Fitting conic sections to scattered data, Computer Vision, Graphics and Image Processing, vol. 9, pp. 56-71, 1979.

[3] E. Boyer: Object Models from Contour Sequences, In Proc. European Conference on Computer Vision, pp. 109-118, 1996.

[4] C.H. Chien and J.K. Aggarwal: Identification of 3D objects from multiple silhouettes using quadtrees/octrees. Computer Vision, Graphics and Image Processing 36, 1986, pp.256-273.

[5] O.D. Faugeras, L. Quan and P. Sturm: Self-calibration of a 1D projective camera and its application to the self-calibration of a 2D projective camera, In Proc. European Conference on Computer Vision, Vol. I, pp. 36-52, Freiburg, Germany, 1998.

[6] O.D. Faugeras and Q.T. Luong: Geometry of Multiple Images, MIT Press, 2001.

[7] M. A. Fischler and R. C. Bolles: Random sample consensus: a paradigm for model fitting with application to image analysis and automated cartography, Communication Association and Computing Machine, 24(6), pp.381-395, 1981

[8] A.W. Fitzgibbon, G. Cross, and A. Zisserman: Automatic 3D Model Construction for Turn-Table Sequences, In 3D Structure from Multiple Images of Large-Scale Environments, European Workshop SMILE'98, Freiburg, Germany, pp. 155-170, 1998.

[9] http://www.robots.ox.ac.uk/vgg/data/

[10] R. Hartley and A. Zisserman: Multiple View Geometry in Computer Vision, Cambridge University Press, 2000.

[11] G. Jiang, H.T. Tsui, L. Quan and A. Zisserman: Single Axis Geometry by Fitting Conics, IEEE Transactions on Pattern Analysis and Machine Intelligence, to appear. It is a summary of the conference papers in CVPR2001 (pp. 293-298, 2001) and ECCV2002 (pp. 537-551).

[12] G. Jiang, L. Quan, and H.T. Tsui: Circular Motion Geometry by Minimal 2 Points in 4 Images, IEEE International Conference on Computer Vision, 2003. 
[13] K.N. Kutulakos and S.M. Seitz: A theory of shape by space carving, IEEE Computer Society Conference on Computer Vision, Vol. 1, pp. 307-314, 1999.

[14] D. Liebowitz and A. Zisserman: Metric rectification for perspective images of planes. IEEE Computer Society Conference on Computer Vision and Pattern Recognition, pp. 482-488, 1998.

[15] Q.-T. Luong and O.D. Faugeras: The Fundamental matrix: theory, algorithms, and stability analysis, International Journal of Computer Vision, pp. 43-76, 1996.

[16] P.R.S. Mendonça, K.-Y.K. Wong, and R. Cipolla: Camera pose estimation and reconstruction from image profiles under circular motion. In Proc. European Conference on Computer Vision, vol. II, pp. 864-877, Dublin, Ireland, Jun 2000. Springer-Verlag.

[17] P.R.S. Mendonça, K.-Y.K. Wong, and R. Cipolla: Epipolar geometry from profiles under circular motion. IEEE Transactions on Pattern Analysis and Machine Intelligence, pp. 604-616, 2001.

[18] W. Niem, and R. Buschmann: Automatic Modelling of 3D Natural Objects from Multiple Views, Yakup Paker and Sylvia Wilbur: Image Processing for Broadcast and Video Production. Workshops in computing series, Springer, Hamburg, 1994.

[19] L. Quan: Invariants of 6 points from 3 uncalibrated images. IEEE Transaction on Pattern Analysis and Machine Intelligence, Vol. 17, No. 1, pp.34-46, 1995.

[20] P.D. Sampson: Fitting conic sections to 'very scattered' data: An iterative refinement of the Bookstein algorithm, Computer Vision, Graphics and Image Processing, vol. 18, pp. 97-108, 1982.

[21] H.S. Sawhney, J. Oliensis, and A.R. Hanson: Image Description and 3-D Reconstruction from Image Trajectories of Rotational Motion. IEEE Transactions on Pattern Analysis and Machine Intelligence, Vol. 15, No. 9, pp. 885-898, 1993.

[22] J. Semple, and G. Kneebone: Algebraic Projective Geometry. Oxford University Press, 1952.

[23] S. Sullivan, and J. Ponce: Automatic model construction, pose estimation, and object recognition from photographs using triangular splines, IEEE Transactions on Pattern Analysis and Machine Intelligence, Vol. 20, No. 10, pp. 1091-1097, 1998.

[24] R. Szeliski: Shape from rotation, IEEE Computer Society Conference on Computer Vision and Pattern Recognition, pp. 625-630, 1991. 
[25] L. Van Gool, M. Proesmans, and A. Zisserman: Planar homologies as a basis for grouping and recognition, In Image and Vision Computing, vol. 16, pp. 21-26, 1998.

[26] T. Vieville and D. Lingrand: Using Specific Displacements to Analyze Motion without Calibration, Int. Journal of Computer Vision, 31(1), pp. 5-29, 1999. 